\title{
Implications and clinical management of myasthenia gravis during pregnancy
}

\author{
Andreea Elena Dumitru', Diana Maria Chitimus², Anca Marina Ciobanu', \\ Nicolae Gica ${ }^{1,3}$, Radu Botezatu ${ }^{1,3}$, Gheorghe Peltecu ${ }^{1,3}$, Anca Maria Panaitescu ${ }^{1,3}$ \\ ${ }^{1}$ Filantropia Clinical Hospital, Bucharest, Romania \\ 2"Carol Davila" Central Military Emergency University Hospital, Bucharest, Romania \\ ${ }^{3}$ Department of Obstetrics and Gynecology, "Carol Davila" University of Medicine and Pharmacy, \\ Bucharest, Romania
}

\begin{abstract}
Myasthenia gravis (MG) is an autoimmune disorder defined by weakness and rapid fatigue of skeletal muscles due to deterioration of neuromuscular junction by autoantibodies targeting either acetylcholine receptors (AchRs) or other molecules such as muscle specific kinase receptors (MuSK). It affects predominantly women in the third decade of life and the effects of pregnancy on the evolution of the disease is variable, usually the aggravation of symptoms occurs during the first trimester or first month postpartum.

The aim of this review is to discuss the clinical management of MG in pregnancy and to highlight the implications of MG on pregnancy and vice-versa, as well as reviewing the available treatment by using documentary analysis.

The findings of this review show a variable clinical course of the disease with remission, worsening or preserving the status quo. MG does not influence the course of pregnancy to a large degree and by careful antenatal care and a multidisciplinary team approach a normal pregnancy and delivery can be achieved.
\end{abstract}

Keywords: myasthenia gravis, pregnancy, weakness, acetylcholinesterase inhibitors

\section{INTRODUCTION}

Myasthenia gravis (MG) is an autoimmune disease, categorized as a type II hypersensitivity causing the production of autoantibodies that block or destroy muscle receptor sites for acetylcholine at the neuromuscular junction of skeletal muscles resulting in fluctuating muscle weakness and muscle fatigue. This prevents nerve impulses from leading to muscle contractions.

With a prevalence of $150-250$ per million, under the age of 40 it is two times more common in women than men, whereas later in life, the incidence is higher in men [1-4].

\section{Clinical aspects}

Regarding the typical progression of the disease, initial weakness often affects only ocular muscles, manifesting as ptosis or diplopia. Within the first two years of presentation, most of the patients will develop generalized weakness which affects the proximal upper and lower extremity muscles. The weakness usually is symmetrical and increases with muscle activity. Although smooth and cardiac muscles are not involved, respiratory muscles are affected in up to $20 \%$ of the cases with AChRs MG and artificial ventilation might be required in myasthenic crisis. Usually, bulbar muscles, involved in speaking, chewing and swallowing, are impaired, therefore dysarthria and dysphagia are also part of the symptomatology [1]. The pathophysiology shows a different distribution of the AChRs antibodies that bound equally to clustered fetal or adult acetylcholine receptors in generalized $\mathrm{MG}$, but only to clustered adult in ocular version [5]. In particular, the fetal AChRs antibodies are important 
due to the placental passage during pregnancy being able to cause either transient neonatal $\mathrm{MG}$ that improves in weeks or a rare and severe condition called fetal arthrogryposis multiplex congenita.

\section{Effects of pregnancy on MG}

Pregnancy has a variable effect on the disease, $41 \%$ of patient will present exacerbation, $29 \%$ remission and $30 \%$ present no change in the course of the disease [6]. Deterioration of the myasthenic symptoms was commonly seen during $1^{\text {st }}$ trimester and $1^{\text {st }}$ month after delivery while recovery of myasthenic symptoms was noted during $2^{\text {nd }}$ and $3^{\text {rd }}$ trimesters probably related to the pregnancy-induced immunosuppression that appears in this period.

The primary causes of exacerbations of $\mathrm{MG}$ during pregnancy consist of:

- hypoventilation due to failing of respiratory muscles and not fully inflated lungs because of the elevation of diaphragm during pregnancy;

- puerperal infections such as endometriosis, mastitis or cystitis;

- drugs;

- thymic abnormalities such as tumors or hyperplastic changes;

- stress of labor and delivery.

The risk of maternal mortality is inversely proportional to the duration of MG before index pregnancy, the highest risk being in the first year after diagnosis of MG and the lowest after 7 years of MG diagnosis [7].

\section{Effects of MG on pregnancy}

MG does not appear to increase the incidence of spontaneous abortion and prematurity, as well as the risk for low birth weight. There has been reported that the premature rupture of amniotic membranes occurs three times more often in myasthenic mothers with an unclear etiology of the rupture, suspecting the effect of medications or polyhydramnios $[8,9]$.

Another concern is preeclampsia that has been outlined in women with MG, yet, no study has demonstrated an increase in the risk of preeclampsia among women with MG. Magnesium sulfate, due to its effect of inhibiting the release of acetylcholine at the neuromuscular junction, is contraindicated in women with MG as treatment in preeclampsia or eclampsia. Maternal deaths have been reported in myasthenic women treated with magnesium sulphate [10]. Moreover, high doses of corticosteroids in myasthenic women who develop preeclampsia may increase fluid retention and aggravate hypertension.

Polyhydramnios resulting from impaired fetal swallowing is a severe complication described in some studies. In extreme cases, high maternal autoantibody titers cause a severe condition known as arthrogryposis multiplex congenita, characterized by lack of fetal movements, contractures in more than two joints, paralysis of fetal diaphragm and increased amniotic fluid volume [11].

Myasthenia gravis patients inherit a susceptibility to other autoimmune diseases such as: rheumatoid arthritis, systemic lupus erythematosus, Hashimoto thyroiditis or scleroderma, therefore thorough assessment should be undertaken.

\section{MANAGEMENT OF PREGNANCY}

\section{Pre-conceptional planning}

Women with MG who intend to get pregnant should be counseled about the potential effects of MG on pregnancy and vice versa. Planning for pregnancy should be established in advance to allow time for the improvement of myasthenic clinical status, thyroid function or drug regimen. Multidisciplinary teams together with the patient should be engaged actively in decision making throughout pregnancy, during delivery and in the postpartum period. Treatment options allowed in pregnancy and possible adverse effects on fetus should be explained in detail. If the disease is well controlled before pregnancy, most of the women will remain stable throughout pregnancy. On the other hand it should be mentioned that symptoms may first appear during pregnancy or, more commonly, during puerperium or in response to paralytic drugs used during general anesthesia.

A pertinent issue regarding treatment of $\mathrm{MG}$ in women is to determine the best timing for thymectomy. This is a standard surgical option for myasthenic patients who have thymic hyperplasia or thymoma [1,3]. It improves clinical outcomes in MG over a 3-year period. The likelihood of worsening of MG during pregnancy, as well as the prospect of neonatal MG are lower in women who have undergone thymectomy compared to women who 
did not [12]. A study from the Medical Birth Registry of Norway concludes that neonates born to mothers with MG who had undergone thymectomy were less prone to develop neonatal MG than those born to mothers without thymectomy (13\% versus $27 \%$ ) [8]. These results suggest that thymectomy has a protective effect against neonatal $\mathrm{MG}$, but there is a delay of at least one year before the therapeutic effects of thymectomy become noticeable. In addition, as thymectomy involves a higher risk-benefit ratio during pregnancy, if the patient conceives it is advised to postpone the intervention after delivery [12].

Antenatal care

In order to provide the best care, the frequency of antenatal visits in pregnant women with MG should be adapted to their clinical status as follows: for symptomatic patients (table 1), visits should be planned every 2 weeks in the first and second trimesters and every week in the third trimester, while pregnant women in clinical remission can be monitored less frequently. Ultrasound evaluation is recommended starting from the first trimester, in order to detect early signs of fetal akinesia such as increased nuchal translucency or hydrops, clubfoot, abnormal position of the limbs. Later in pregnancy it is important to monitor fetal movements or breathing motion and evidence of polyhydramnios after 25 weeks of gestation [13].

Screening for asymptomatic bacteriuria or other infections and prompt treatment should be established as it might worsen and all women should undergo periodic detailed assessment of baseline motor strength, respiratory and cardiovascular status as well as thyroid function tests.

TABLE 1. Evaluation of pregnancy associated with MG

\begin{tabular}{|l|l|l|}
\hline Maternal & Fetal & Neonatal \\
\hline $\begin{array}{l}\text { Pulmonary function } \\
\text { test }\end{array}$ & Ultrasound & Observation \\
\hline Thyroid function test & Biophysical profile & Pulsoximetry \\
\hline EKG & Amniotic fluid index & Respiratory rate \\
\hline Neurologic evaluation & Body movements & Ability to feed \\
\hline
\end{tabular}

\section{Therapy during pregnancy}

Main recommendations for pregnant women with MG are to avoid unnecessary fatigue, emotional stress and lack of sleep, to lay down several times during the day or to rest the eyes by closing them for a few minutes regularly, combined with a balanced diet containing food high in potassium.

The symptomatic treatment in MG includes anticholinesterase agents (pyridostigmine and neostigmine) at doses adjusted depending on the renal clearance, expanded maternal blood volume and delayed gastric emptying [14].

In acute exacerbations, immunosuppressants such as corticosteroids, intravenous immunoglobulin or plasma exchange can be considered. In patients with moderate to severe generalized weakness with poor response to anticholinesterase drugs, long-term administration of corticosteroids is an optimal solution. Corticosteroid therapy is effective, but remission appears to be preserved only during continuous therapy and withdrawal may cause myasthenic exacerbations; for these reasons, it should be maintained throughout pregnancy and the postpartum period. Corticosteroid therapy appears to be safe during pregnancy although it was reported a slight raise in the incidence of cleft palate $(1 \%)[15,16]$. Also, special attention should be offered to infants of mothers with high dose of steroids administered during the third trimester due to potential fetal adrenal suppression and cortisone substitution can be considered in the neonatal period [17].

Azathioprine is a useful adjunct to steroids in patients who cannot tolerate or fail to respond to prednisone. Azathioprine and cyclosporine may be used particularly in transplant recipients and patients with autoimmune diseases other than MG, being relatively safe. Azathioprine can cross the placenta, but because the fetal liver lacks the enzyme that converts the drug into its active metabolites the fetus is protected from any teratogenic effect early in pregnancy [18]. However, high dosage of cyclosporine and azathioprine has been linked to spontaneous abortion, preterm labor, low birth weight and hematopoietic cells suppression $[19,20]$. They should be reserved for pregnant women who are poorly controlled, cannot tolerate glucocorticoids or have significant comorbidities that may worsen with glucocorticoids.

During lactation it is recommended to replace anticholinesterase drugs and other immunosuppressive therapy as azathioprine and cyclosporine with corticosteroids, having the least side effects on newborn although all of these drugs could be found in breast milk [21]. 
Mycophenolate mofetil and methotrexate should be avoided in pregnancy, current data suggesting teratogenic effects such as: short fingers and toenails, cleft palate and lip and agenesis of corpus callosum.

Severe exacerbations or myasthenic crisis require either third line therapy which involves reduction in the maternal autoantibodies by plasmapheresis or fourth line treatment- blockage of the effect of autoantibodies by intravenous immunoglobulin.

\section{Labor and delivery}

Vaginal delivery is considered safe in pregnant women with MG. Cesarian delivery should be performed only for obstetrical indications as surgery is often linked with worsening of MG and can even trigger myasthenic crisis. Being composed of smooth muscle, the uterus is not affected by disease process and its contractility is maintained, thus the first stage of labor is not affected, but the second stage of labor concerns striated muscles, so it might be prolonged and the patient may require assistance in the form of operative delivery (forceps delivery and/or vacuum extraction).

During labor, epidural analgesia should be used to alleviate pain, but the use of sedatives and opioids should be avoided as these may precipitate respiratory depression. Local anesthetic agents should be avoided as these can block neuromuscular transmission.

\section{Postpartum management and neonatal outcome}

Glucocorticoids are poorly excreted in breast milk and their use during lactation is considered safe. Breastfeeding is not recommended for patients with MG taking azathioprine, cyclosporine and methotrexate. Anticholinesterase drugs are found in breast milk in low levels and are therefore granted safe in lactation unless high doses are required.

There are three types of neonatal MG: transient neonatal myasthenia occurs in 10 to $20 \%$ of babies born to mothers with MG and disappears after a few weeks, congenital myasthenia, occurs when genes are present from both parents and it represents the rarest form and juvenile myasthenia gravis is more common in females [22].

During pregnancy, due to the transplacental passage of Ig G AchRs antibodies transient neonatal
MG can develop in 10-20\% of infants. Therefore, symptoms of respiratory distress, difficulties in feeding and sucking, ptosis, feeble cry, facial paresis and hypotonia appear within $48 \mathrm{~h}$ of birth and may persist for up to 3 months [14]. About $2 \%$ of infants can develop a rare condition known as fetal arthrogryposis multiplex congenita due to the passage of maternal antibodies directed against the fetal-type of Ach Rs [23,24]. The lack of movement and abnormal position with fixed flexion or extension deformities in fetal joints can be diagnosed by prenatal ultrasound (Figure 1). These receptors found in fetal muscles are replaced by the adulttype by 33 weeks of gestation and mothers carrying this type are mostly asymptomatic with the possibility of developing the disease later by producing also antibodies against the adult-type receptors [25].

The severity and the duration of MG in mothers do not correlate with the risk of developing neonatal MG. Among women with a child already affected by transient neonatal MG, the risk of recurrence in subsequent pregnancies is $75 \%$ [26].

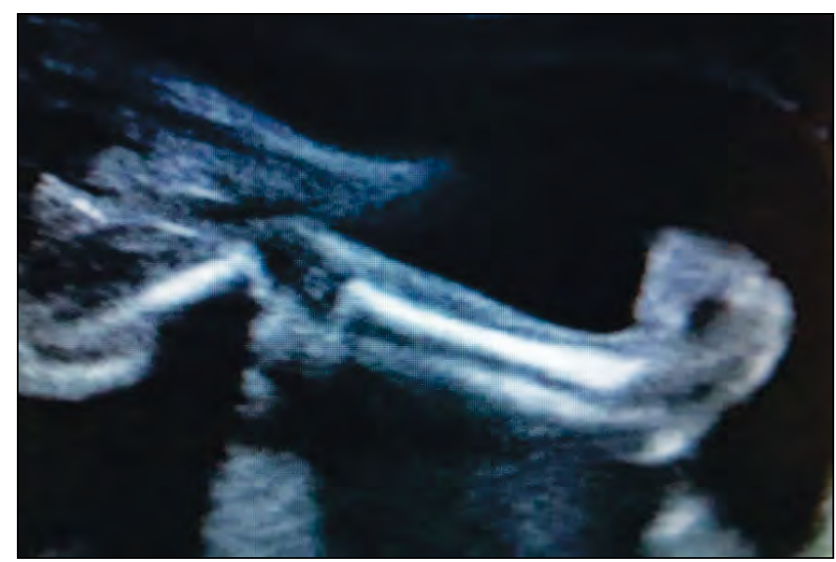

FIGURE 1. Fetal arthrogryposis multiplex congenita with flexion deformity to the knee and talipes

\section{CONCLUSIONS}

The clinical course of MG in pregnancy is variable, with chances of remission, worsening or unchanged evolution. Pre-pregnancy counseling should be given to all women with MG and more frequent antenatal visits should be planned. To reduce the risk of fetal harm, it is recommended to perform thymectomy one year prior to conception. The treatment of MG during pregnancy should be determined having in mind both benefits and fetal 
risks. While pyridostigmine alone may be acceptable for women with mild isolated ocular symptoms, immunosuppression is often needed for more severe symptoms. Steroids are the immunosuppressant drugs of choice and should be used in lowest effective dose. Patients who are intolerant or re- fractory may need azathioprine or cyclosporine. There should be a careful observation of all newborn for at least $72 \mathrm{~h}$ due to $20 \%$ risk of transient neonatal MG and intensive care facilities should be quickly accessible in case of need.

Conflict of interest: none declared Financial support: none declared

\section{REFERENCES}

1. Gilhus NE. Myasthenia gravis. N Engl J Med. 2016;375:2570-81.

2. Carr AS, Cardwell CR, McCarron PO, McConville J. A systematic review of population based epidemiological studies in myasthenia gravis. BMC Neurol. 2010;10:46.

3. Ferrero S, Esposito F, Biamonti M, Bentivoglio G, Ragni N. Myasthenia gravis during pregnancy. Expert Rev Neurother. 2008;8:979-88.

4. Ferrero S, Pretta S, Nicoletti A, Petrera P, Ragni N. Myasthenia gravis: Management issues during pregnancy. Eur J Obstet Gynecol Reprod Biol. 2005;121:129-38.

5. Jacob S, Viegas S, Leite Ml et al. Presence and pathogenic relevance of antibodies to clustered acetylcholine receptor in ocular and generalized myasthenia gravis. Arch. Neurol. 2012;69:994-1001.

6. Plauché WC. Myasthenia gravis in mothers and their newborns. Clin Obstet Gynecol 1991;34:82.

7. Chaudhry SA, Vignarajah B, Koren G. Myasthenia gravis during pregnancy. Can Fam Physician. 2012;58:1346-9.

8. Hoff JM, Daltveit AK, Gilhus NE. Myasthenia gravis in pregnancy and birth: Identifying risk factors, optimising care. Eur J Neurol. 2007;14:38-43.

9. Hoff JM, Daltveit AK, Gilhus NE. Myasthenia gravis: Consequences for pregnancy, delivery, and the newborn. Neurology. 2003; 61:1362-6.

10. Cohen BA, London RS, Goldstein PJ. Myasthenia gravis and preeclampsia. Obstet Gynecol 1976;48(Suppl. 1):35S-7S.

11. Verspyck $E$, Mandelbrot $L$, Dommergues $M$, Huon $C$, Woimant $F$, Baumann C, Vernet- Der Garabedran B. Myasthenia gravis with polyhydramnios in the fetus of an asymptomatic mother. Prenat Diagn. 1993;13:539-42.

12. Wolfe GI, Kaminski HJ, Aban IB, Minisman G, Kuo HC, Marx A, et al. Randomized trial of thymectomy in myasthenia gravis. N Engl J Med. 2016;375:511-22.

13. Skaria $P$, Dahl A, Ahmed A Arthrogryposis multiplex congenita in utero: Radiologic and pathologic findings. J Matern Fetal Neonatal Med. 2019;32(3):502-511.

14. Stafford IP, Dildy GA. Myasthenia gravis and pregnancy. Clin Obstet Gynecol 2005;48:48.
15. Fraser FC, Sajoo A. Teratogenic potential of corticosteroids in humans. Teratology 1995;51:45-6.

16. Rodriguez-Pinilla E, Martinez-Frias ML. Corticosteroids during pregnancy and oral clefts: A case-control study. Teratology 1998;58:2-5

17. Park-Wyllie L, Mazzotta P, Pastuszak A et al. Birth defects after maternal exposure to corticosteroids: prospective cohort study and meta-analysis of epidemiological studies. Teratology. 2000; 62:385-392.

18. Janssen NM, Genta MS. The effects of immunosuppressive and antiinflammatory medications on fertility, pregnancy, and lactation. Arch Intern Med. 2000;160:610-9.

19. Ramsey-Goldman R, Schilling E. Immunosuppressive drug use during pregnancy. Rheum Dis Clin North Am. 1997;23:149.

20. Haugen G, Fauchald P, Sødal $G$ et al. Pregnancy outcome in renal allograft recipients in Norway. The importance of immunosuppressive drug regimen and health status before pregnancy. Acta Obstet Gynecol Scand. 1994;73:541.

21. Batocchi AP, Majolini L, Evoli A, et al. Course and treatment of myasthenia gravis during pregnancy. Neurology. 1999;52:447-452.

22. Rudd K, Kocisko D (2013). Pediatric Nursing: The Critical Components of Nursing Care. F.A. Davis. ISBN 978-0-8036-4053-5. Archived from the original on 3 June 2016.

23. Hoff JM, Daltveit AK, Gilhus NE. Artrogryposis multiplex congenita - a rare fetal condition caused by maternal myasthenia gravis. Acta

24. Vincent A, McConville J, Farrugia ME et al. Antibodies in myasthenia gravis and related disorders. Ann N Y Acad Sci. 2003;998:324-335.

25. Anca Maria Panaitescu, Kypros Nicolaides Maternal autoimmune Medicine 2018;31(13):1798-1806.

26. Lu CH, Liou CM, Chen YS, Hung CJ, Tho HS. Anesthetic management in myasthenic parturient. Anaesthesiol Sin. 1992;30:193-7. Neurol Scand Suppl. 2006;183:26-27. disorders and fetal defects, The Journal of Maternal-Fetal \& Neonatal 